\title{
Near-deterministic preparation of a single atom in an optical microtrap
}

\author{
T. Grünzweig, A. Hilliard, M. McGovern and M. F. Andersen *
}

\begin{abstract}
Neutral atoms stored in optical traps are strong candidates for a physical realization of a quantum logic device ${ }^{1,2}$. Far off-resonance optical traps provide conservative potentials and excellent isolation from the environment, and they may be arranged to produce arbitrary arrays of traps, where each trap is occupied by a single atom that can be individually addressed $^{3-6}$. At present, significant effort is being expended on developing two-qubit gates based on coupling individual Rydberg atoms in adjacent optical microtraps ${ }^{7-9}$. A major challenge associated with this approach is the reliable generation of single-atom occupancy in each trap, as the loading efficiency in the past experiments has been limited to $50 \%$ (refs $4,7,8,10-12$ ). Here we report a loading efficiency of $82.7 \%$ in an optical microtrap. We achieve this by manipulating the collisions between pairs of trapped atoms through tailored optical fields and directly observing the resulting single atoms in the trap.
\end{abstract}

Deterministic control of single neutral atoms is a long-standing goal in atomic physics. Not only would it represent a milestone in scientists' ability to control the microscopic world, but also because it would enable a neutral-atom-based quantum logic device ${ }^{7-9}$. Two approaches have successfully led to direct observation of subPoissonian number distributions of atoms in optical microtraps, without consecutive atom sorting ${ }^{13}$. In the first, the Mott insulator transition of a Bose-Einstein condensate provides an efficient route for high occupancy of individual atoms in optical lattices where atoms can tunnel between adjacent lattice sites ${ }^{14-17}$. The second approach, which may be applied in arbitrary geometries ${ }^{18,19}$, is to employ light-assisted collisions ${ }^{4,10-12}$. This method makes use of the change in the atom-atom interaction that arises when light drives one of the atoms undergoing a collision to the electronic excited state. In the case of light with a frequency below resonance (red detuned), the atom pair is excited to an attractive potential leading to the atoms forming a molecule and/or gaining a large amount of kinetic energy. In each case, both atoms are lost, leading to a maximal $50 \%$ chance of ending with one atom in the trap, depending on whether the initial atom number is even or odd ${ }^{10,12}$. However, a process where only one atom is lost as a result of a two-body collision would lead to deterministic preparation of a single atom in a given site. In the past, it has been shown that various forms of collisional trap loss can be suppressed by the application of optical control fields, and in particular, the use of blue-detuned light to effect so-called optical shielding ${ }^{20,21}$.

In this Letter, we study light-assisted collisions at the single-event level. We prepare individual pairs of atoms in an optical microtrap and expose them to near-resonant light. We directly observe that light-assisted collisions between these atoms can lead to only one atom being lost. By choice of the frequency and intensity of the collision-inducing light we can control the energy released in an inelastic light-assisted collision ${ }^{22}$. By releasing only enough energy in each collision for one atom to escape the trap, the probability of one atom being lost can dominate over the two-atom-loss mechanisms observed in refs 4,10-12, thereby yielding a high loading efficiency. In the experiment, we use ${ }^{85} \mathrm{Rb}$ and give the first images of a single atom of this isotope (see Fig. 1a).

The microscopic dipole trap is formed by focusing a far offresonant laser beam with a high-numerical-aperture aspheric lens mounted inside a vacuum chamber. Figure $1 \mathrm{~b}$ is a schematic of the set-up. The high-numerical-aperture lens also collects light scattered by the trapped atom(s) and forms a high-resolution image on a low-light-sensitive camera ${ }^{11}$ (see Supplementary Information). To induce light-assisted collisions and image the sample, we illuminate the atoms with a quasi-resonant, retro-reflected probe beam that propagates close to orthogonal to the dipole trap beam. Each experiment begins by loading approximately 50 atoms from a magneto-optical trap (MOT) into the optical microtrap. We then optically pump the atoms into the $F=2$ ground state using the MOT cooling beams, and induce light-assisted collisions by turning on a probe beam on the $\mathrm{D} 1$ line of ${ }^{85} \mathrm{Rb}$ with a frequency that is detuned by an amount $\delta_{\mathrm{c}} \approx 97 \mathrm{MHz}$ above the $F=2$ to $F^{\prime}=3$ resonance for atoms at the centre of the dipole trap. Figure 1c shows how the atomic energy levels for the D1 line are shifted by the dipole trap ${ }^{23}$. Note that $h \delta_{\mathrm{c}}$ is approximately equal to the depth of the dipole trap, calculated to be $U_{0}=h \times 102 \mathrm{MHz}\left(4.9 \mathrm{mK} / K_{\mathrm{B}}\right)$. The cooling beams for the MOT remain on during collisions, but with a frequency shifted to the free-space $F=3$ to $F^{\prime}=4$ resonance. Owing to the light shift induced by the dipole trap, the MOT cooling beams' frequency is close to resonance on the D2 $F=3$ to $F^{\prime}=3$ transition for atoms at the centre of the trap. As such, the cooling beams serve to optically pump the atoms back into the $F=2$ ground state for atoms at the centre of the trap. They also restore the final temperature of a single trapped atom of $450 \pm 50 \mu \mathrm{K}$, as measured by the release and recapture technique ${ }^{24}$.

Inelastic light-assisted collisions induced by blue-detuned light release an amount of energy limited by the detuning to $h \delta$ from the single-atom resonance. This process occurs when an atom pair enters on the $\mathbf{S}+\mathbf{S}$ interatomic potential and exits on $\mathbf{S}+\mathbf{P}$; an example of such a process is illustrated in Fig. 2a (ref. 25). A collision is most likely to occur at the centre of the trap, where the density is highest. With our choice of detuning, this means $\delta \approx \delta_{\mathrm{c}} \approx U_{0} / h$. After such a collision, the atoms will have only enough energy for one of them to escape the trap. This process can therefore directly induce one-atom loss, if the pair's initial thermal centre-of-mass motion makes them share the gained energy unevenly, but it cannot cause two-atom loss. Figure $2 \mathrm{a}$ also illustrates a second process known as optical shielding ${ }^{20}$ : at high intensities, the atom pair can transfer between the $\mathbf{S}+\mathbf{S}$ and $\mathbf{S}+\mathbf{P}$ states through adiabatic passage each time the interatomic separation crosses $R_{\mathrm{c}}$, thereby effecting an elastic collision if no spontaneous emission event occurs. When an 

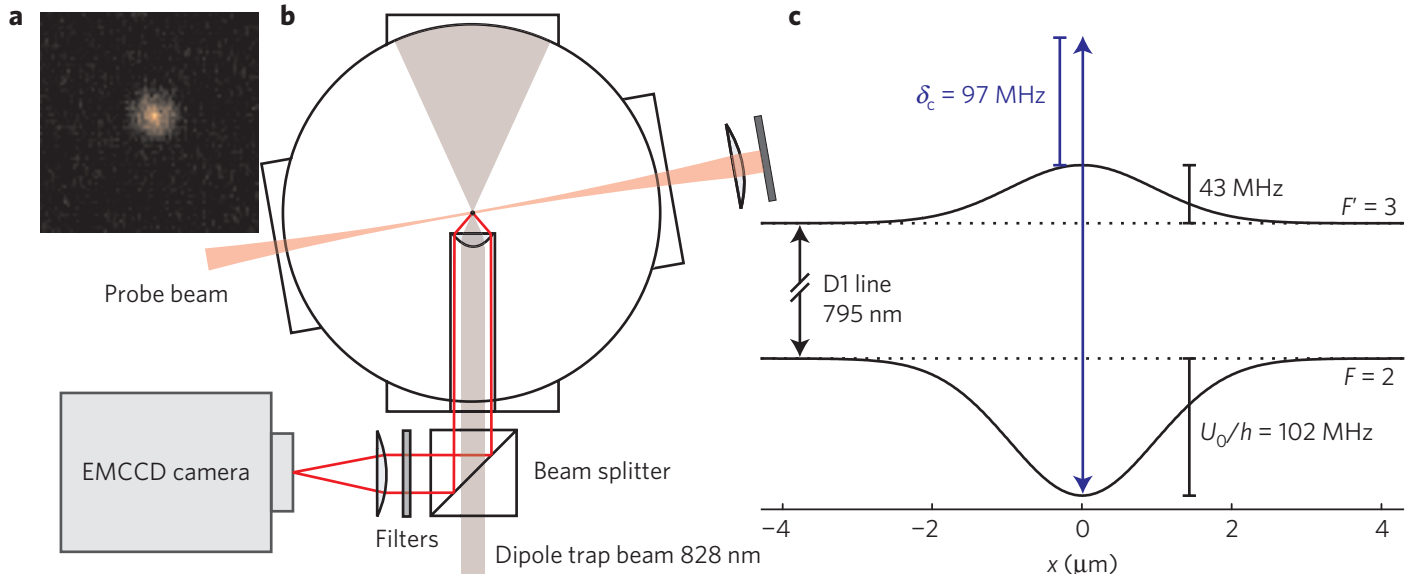

Figure 1 | Experimental schematics. a, An image of a single ${ }^{85} \mathrm{Rb}$ atom. $\mathbf{b}$, A schematic of the experiment. The high-numerical-aperture lens focuses the dipole trap beam to form an optical microtrap at the centre of the vacuum chamber. This lens is the first element in an infinity-corrected microscope that images fluorescence from a trapped atom onto an electron-multiplying charge-coupled device (EMCCD). c, Calculated spatially dependent light shifts of the $F=2$ to $F^{\prime}=3 \mathrm{D} 1$ transition along the tight dimension of the trap for $36 \mathrm{~mW}$ of light at $828 \mathrm{~nm}$ and a waist of $w_{0}=1.8 \mu \mathrm{m}$. The blue double-headed arrow indicates the frequency of the collision light. At the centre of the trap, the light is blue detuned by an amount $\delta_{\mathrm{c}}$.
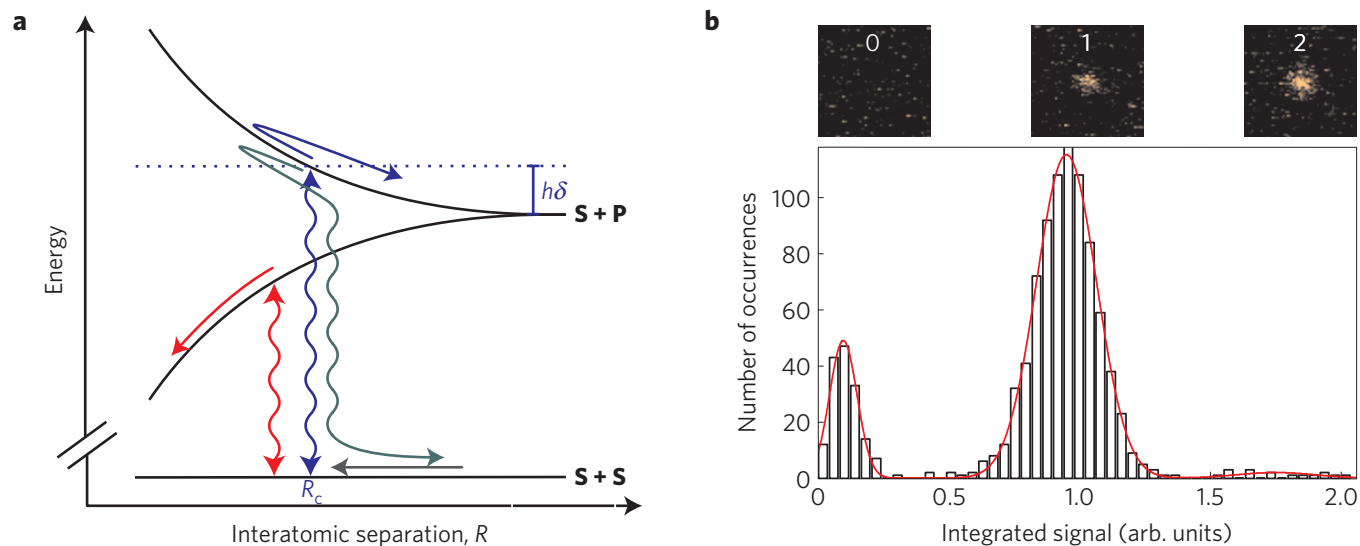

Figure 2 | Deterministic preparation of single atoms. a, Simple one-dimensional model of light-assisted collisions. Grey arrow: two atoms in their electronic ground states approach each other. Red arrow: in the presence of red-detuned light, the atom pair is excited to an attractive atom-atom potential, leading to the pair loss observed in refs 4,10-12. In the presence of blue-detuned light, the atom pair is excited to a repulsive potential. Blue arrow: an inelastic collision ensues, leading to the atom pair gaining a maximal $h \delta$ in energy, followed by decay to the $\mathbf{S}+\mathbf{S}$ state ${ }^{25}$. Green arrow: optical shielding. When the light induces a transition each time the interatomic separation crosses $R_{\mathrm{c}}$ the collision is elastic ${ }^{20}$. $\mathbf{b}$, Top, from left to right: images of zero, one and two atoms remaining in the trap after applying a collision pulse. Bottom, histogram of the integrated fluorescence for 1,000 realizations showing resolvable peaks for the three possible outcomes, namely zero, one or two atoms. The red line is a guide to the eye. In $82.7 \%$ of the outcomes we obtain a single atom.

atom is not ejected from the trap directly by the inelastic collision, these elastic collisions may redistribute the gained energy between the atoms and potentially induce the loss of a single atom before the interaction with the cooling light has significantly reduced their energy or a second inelastic collision occurs.

Figure $2 \mathrm{~b}$ shows a histogram of the integrated fluorescence for 1,000 realizations of this experiment for a $300 \mathrm{~ms}$ collision pulse. The three clearly resolved peaks correspond to the outcome of the experiment being zero, one or two atoms remaining in the microtrap. The largest peak, comprising $82.7 \pm 1.2 \%$ of the realizations, corresponds to the one-atom case. $15.7 \%$ of the realizations resulted in zero atoms left in the trap, and the remaining $1.6 \%$ resulted in two atoms. Extending the probe pulse beyond $300 \mathrm{~ms}$ eliminates the possibility of obtaining two atoms in the trap, but also leads to a reduction in the probability of obtaining only one atom.

To verify our interpretation that the high preparation efficiency is obtained by collision-induced one-atom loss, we prepare pairs of trapped atoms and directly observe their evolution. First, a $100 \mathrm{~ms}$ preliminary collision pulse produces a low number of atoms in the trap. The resulting atomic sample is imaged before and after a second collision pulse of a variable duration. From 400 experimental runs for each collision pulse duration, we select only realizations with two atoms in the first image $(\sim 25 \%$ of the realizations). For this group, we plot in Fig. 3 the fraction of cases that end with zero, one or two atoms remaining in the trap, for the same collision pulse detuning and power used for Fig. 2. The data show that the dominant decay mechanism is one-atom loss resulting from two-body collisions. The single-atom lifetime is $3.5 \mathrm{~s}$, so loss resulting from this mechanism is negligible on the timescale of two-atom decay. By fitting the data with a model that assumes that a pair of atoms decays exponentially to either zero or one remaining atoms, we obtain the probabilities for these outcomes to be $p(0 \mid 2)=0.14$ and $p(1 \mid 2)=0.86$ respectively. The model is presented in the Supplementary Information. Previous experiments with small samples of caesium atoms trapped in a high-gradient 


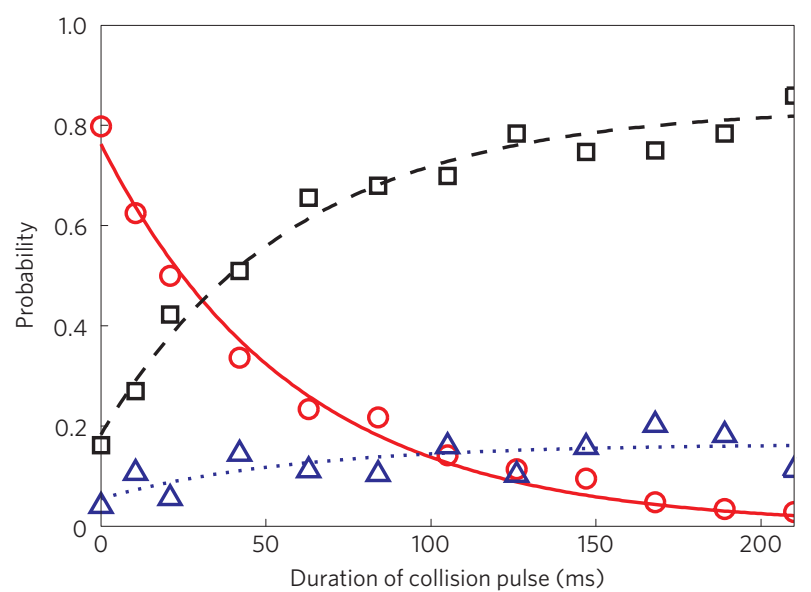

Figure 3 | Time evolution of pairs of atoms. The red circles show the probability of pair survival as a function of the collision pulse duration. The black squares and blue triangles show the probabilities of obtaining one or zero atoms respectively after the collision pulse. The curves are a fit of the measured probabilities of two (red solid line), one (black dashed line) and zero (blue dotted line) atoms (see Supplementary Information).

MOT identified a similar process, where only one atom was lost as a result of a light-assisted collision ${ }^{26}$. The existence of a similar process induced by red-detuned light and with lower probability in an optical dipole trap was predicted in ref. 27.

Figure 4 shows how the probability of obtaining one atom in the trap evolves as a function of the collision pulse duration. The data exhibit an initial rapid growth as the light-assisted collisions produce single atoms in the trap. The one-atom probability then decays slowly with the single-atom lifetime. The peak probability is consistent with Fig. $2 \mathrm{~b}$ and occurs for pulse durations around $300 \mathrm{~ms}$ having started with approximately 50 atoms in the trap. The probability to end with one atom, starting with infinitely many atoms in the trap, can be shown to be $p(1 \mid \infty)=1 /(2-$ $p(1 \mid 2))=0.87$ in the absence of single-atom decay. To account for the finite single-atom lifetime, we use the parameters deduced from Fig. 3 and the measured single-atom lifetime in a Monte Carlo simulation (see Supplementary Information). The result shown in Fig. 4 is in good agreement with our measurements, leading to the conclusion that the loading efficiency can be improved even further by improving the single-atom lifetime; for example, through improving the vacuum.

We have demonstrated a simple and efficient method to generate a high level of single-atom occupancy in optical microtraps. By direct observation of the outcome of individual collision events, we have shown that high preparation efficiency is achieved by tuning parameters such that inelastic light-assisted collisions result in only one atom being lost. We have trapped and imaged single ${ }^{85} \mathrm{Rb}$ atoms for the first time. This isotope is of special interest because of its accessible Feshbach resonances ${ }^{28}$ that can be used to tune interactions to entangle pairs of atom. Our work provides a route to extend present work on quantum logic devices ${ }^{7,8}$ to considerably more complicated systems.

\section{Methods}

Microtrap. A $500 \mathrm{~ms}$ MOT stage initially cools and traps a cloud of approximately $10^{5}$ atoms. Next, a $150 \mathrm{~ms}$ compressed MOT stage ${ }^{29}$ increases the sample density, whereupon the magnetic field is extinguished and the dipole trap beam is switched on. The loading into the dipole trap takes place over $5 \mathrm{~ms}$ with the MOT beams detuned to effect polarization gradient cooling ${ }^{30}$. This procedure loads approximately 50 atoms into the microtrap.

The microtrap is generated using light $828 \mathrm{~nm}$ and a power of $36 \mathrm{~mW}$ (measured before the chamber). A high-numerical-aperture lens ( $\mathrm{NA}=0.55$ ), with a working distance of $2.92 \mathrm{~mm}$, focuses the trap beam to form the microtrap. This

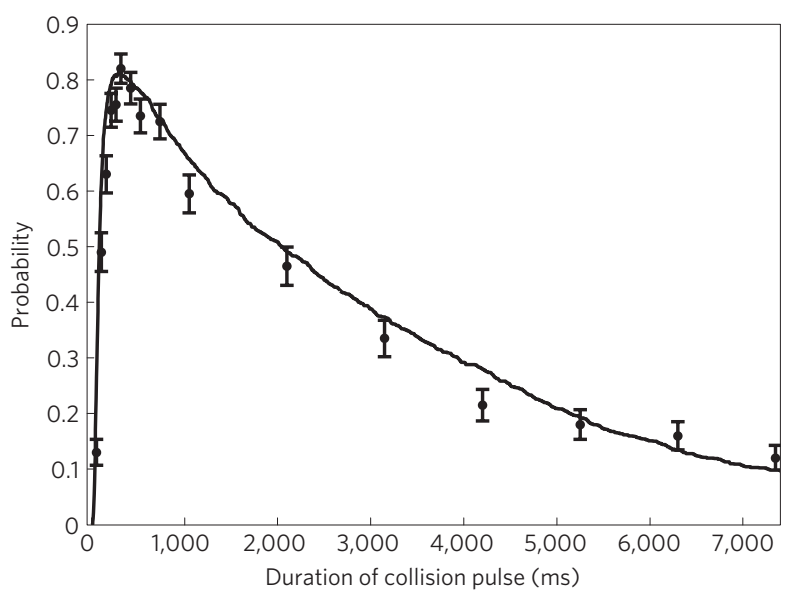

Figure 4 | Single-atom probability as a function of the duration of the collision pulse. Measurement with error bars that represent the 95\% confidence estimate of the error resulting from binomial statistics of the 200 realizations used per data point. The line is a Monte Carlo simulation of the model described in the Supplementary Information, with model parameters deduced from Fig. 3.

lens is mounted inside the vacuum chamber, such that its focus is formed inside the compressed-MOT cloud. At the focal point, the trapping beam has a $1 / e^{2}$ radius of $w_{0}=1.8 \mu \mathrm{m}$. Both the vacuum windows and the high-numerical-aperture lens are anti-reflection coated on each surface.

The calculated dipole-trap-induced light shifts have been confirmed experimentally. Parametric excitation of single atoms in the trap produced measured trap frequencies that were consistent with the calculated values. We have also measured the fluorescence spectrum on the $F=2$ to $F^{\prime}=3$ transition on the D1 line of a single trapped atom for the presented trap parameters. These measurements were in very good agreement with the calculation presented in Fig. 1c.

Light-assisted collisions. We induce light-assisted collisions of atoms in the $F=2$ ground state using light that is blue detuned by an amount $\delta$ from the $F=2$ to $F^{\prime}=3$ transition on the D1 line, such that we can limit the amount of energy released to $h \delta . h \delta$ must be smaller than twice the trap depth, yet to selectively excite the repulsive atom-atom potential, $\delta$ must be significantly larger than the natural linewidth $\Gamma$. This constraint favours deep traps. We aim to avoid light-assisted collisions of atoms in the $F=3$ ground state, either by the D1 light, or the MOT cooling beams, as both can release significantly more energy than twice the trap depth and therefore lead to two-atom loss.

We obtain single-atom loading efficiencies greater than $50 \%$ for a large range of the accessible experimental parameters.

The behaviour with respect to the blue detuning of the D1 $F=2$ to $F^{\prime}=3$ transition is robust, with loading efficiencies exceeding $50 \%$ observed for $25 \mathrm{MHz} \leq \delta_{\mathrm{c}} \leq 105 \mathrm{MHz}$. Although we obtain above $50 \%$ loading efficiency for most intensities accessible in our experiment, the optimal value depends on the detuning. As $\delta_{\mathrm{c}}$ is decreased, the optical power that leads to optimal loading efficiency gradually shifts to lower values.

During collision pulses we keep the MOT cooling beams on but tuned close to the $F=3$ to $F^{\prime}=3 \mathrm{D} 2$ transition for atoms at the bottom of the trap. This optically pumps atoms that spontaneously decay into the $F=3$ ground state back into the $F=2$ ground state where the preferred light-assisted collisions can occur. However, away from the centre of the trap, the light shift induced by the dipole trap decreases, and the atoms experience a frequency closer to the free-space $F=3$ to $F^{\prime}=4$ transition. As such, as an atom moves to the edge of the trap, there will be a transition from predominantly optical pumping on the $F=3$ to $F^{\prime}=3$ transition, to standard laser cooling on the $F=3$ to $F^{\prime}=4$ transition. The measured single-atom temperature of $450 \mu \mathrm{K}$ is above typical sub-Doppler cooling temperatures, because the MOT cooling beams are optimized to produce high single-atom loading, rather than low temperature.

Using our scheme we obtained high loading efficiencies for a trap depth of $57 \mathrm{MHz}$ as well, indicating that the exact trap depth is not a crucial parameter.

Received 21 May 2010; accepted 10 August 2010; published online 26 September 2010

\section{References}

1. Feynman, R. Simulating physics with computers. Int. J. Theor. Phys. 21, 467-488 (1982).

2. DiVincenzo, D. P. Quantum computation. Science 270, 255-261 (1995). 
3. Schrader, D. et al. Neutral atom quantum register. Phys. Rev. Lett. 93, 150501 (2004)

4. Nelson, K. D., Li, X. \& Weiss, D. S. Imaging single atoms in a three-dimensional array. Nature Phys. 3, 556-560 (2007).

5. Karski, M. et al. Nearest-neighbor detection of atoms in a $1 \mathrm{D}$ optical lattice by fluorescence imaging. Phys. Rev. Lett. 102, 053001 (2009).

6. Dumke, R. et al. Micro-optical realization of arrays of selectively addressable dipole traps: A scalable configuration for quantum computation with atomic qubits. Phys. Rev. Lett. 89, 097903 (2002).

7. Gaetan, A. et al. Observation of collective excitation of two individual atoms in the Rydberg blockade regime. Nature Phys. 5, 115-118 (2009).

8. Urban, E. et al. Observation of Rydberg blockade between two atoms. Nature Phys. 5, 110-114 (2009).

9. Zuo, Z. et al. Single atom Rydberg excitation in a small dipole trap. Opt. Express 17, 22898-22905 (2009).

10. DePue, M. T., McCormick, C., Winoto, S. L., Oliver, S. \& Weiss, D. S. Unity occupation of sites in a 3D optical lattice. Phys. Rev. Lett. 82, 2262-2265 (1999).

11. Schlosser, N., Reymond, G., Protsenko, I. \& Grangier, P. Sub-Poissonian loading of single atoms in a microscopic dipole trap. Nature 411, 1024-1027 (2001)

12. Schlosser, N., Reymond, G. \& Grangier, P. Collisional blockade in microscopic optical dipole traps. Phys. Rev. Lett. 89, 023005 (2002).

13. Miroshnychenko, Y. et al. Quantum engineering: An atom-sorting machine. Nature 442, 151-151 (2006).

14. Jaksch, D., Bruder, C., Cirac, J. I., Gardiner, C. W. \& Zoller, P. Cold bosonic atoms in optical lattices. Phys. Rev. Lett. 81, 3108-3111 (1998).

15. Bakr, W. S. et al. Probing the superfluid-to-Mott-insulator transition at the single-atom level. Science 329, 547-550 (2010).

16. Esteve, J., Gross, C., Weller, A., Giovanazzi, S. \& Oberthaler, M. K. Squeezing and entanglement in a Bose-Einstein condensate. Nature 455, 1216-1219 (2008).

17. Itah, A. et al. Direct observation of a sub-Poissonian number distribution of atoms in an optical lattice. Phys. Rev. Lett. 104, 113001 (2010).

18. Beugnon, J. et al. Two-dimensional transport and transfer of a single atomic qubit in optical tweezers. Nature Phys. 3, 696-699 (2007).

19. Bergamini, S. et al. Holographic generation of microtrap arrays for single atoms J. Opt. Soc. Am. B 21, 1889-1894 (2004).

20. Bali, S., Hoffmann, D. \& Walker, T. Novel intensity dependence of ultracold collisions involving repulsive states. Europhys. Lett. 27, 273-277 (1994).
21. Marcassa, L. et al. Optical suppression of photoassociative ionization in a magneto-optical trap. Phys. Rev. Lett. 73, 1911-1914 (1994).

22. Weiner, J. Cold and Ultracold Collisions in Quantum Microscopic and Mesoscopic Systems (Cambridge Univ. Press, 2003).

23. Grimm, R., Weidemüller, M. \& Ovchinnikov, Y. B. Optical dipole traps for neutral atoms. Adv. At. Mol. Opt. Phys. 42, 95-170 (2000).

24. Tuchendler, C., Lance, A. M., Browaeys, A., Sortais, Y. R. P. \& Grangier, P. Energy distribution and cooling of a single atom in an optical tweezer. Phys. Rev. A 78, 033425 (2008).

25. Hoffmann, D., Bali, S. \& Walker, T. Trap-depth measurement using ultra-cold collisions. Phys. Rev. A 54, R1030-R1033 (1996).

26. Ueberholz, B., Kuhr, S., Frese, D., Gomer, V. \& Meschede, D. Cold collisions in a high-gradient magneto-optical trap. J. Phys. B 35, 4899-4914 (2002).

27. Forster, L. et al. Number-triggered loading and collisional redistribution of neutral atoms in a standing wave dipole trap. New J. Phys. 8, 259 (2006).

28. Cornish, S. L., Claussen, N. R., Roberts, J. L., Cornell, E. A. \& Wieman, C. E. Stable ${ }^{85} \mathrm{Rb}$ Bose-Einstein condensates with widely tunable interactions. Phys. Rev. Lett. 85, 1795-1798 (2000).

29. Cornell, E. A. \& Wieman, C. E. Nobel lecture: Bose-Einstein condensation in a dilute gas, the first 70 years and some recent experiments. Rev. Mod. Phys. 74, 875-893 (2002).

30. Phillips, W. D. Nobel lecture: Laser cooling and trapping of neutral atoms Rev. Mod. Phys. 70, 721-741 (1998).

\section{Acknowledgements}

We gratefully acknowledge T. G. Walker for proposing the role of optical shielding and for his invaluable help in understanding the process. We also wish to thank E. Tiesinga for his helpful comments on the manuscript. This work is supported by NZ-FRST Contract No. NERF-UOOX0703 and UORG.

\section{Author contributions}

All authors contributed to all aspects of this work.

\section{Additional information}

The authors declare no competing financial interests. Supplementary information accompanies this paper on www.nature.com/naturephysics. Reprints and permissions information is available online at http://npg.nature.com/reprintsandpermissions. Correspondence and requests for materials should be addressed to M.F.A. 